\title{
If
}

HSOA Journal of Dentistry: Oral Health \& Cosmesis

\section{Occlusal Trauma May Be a Factor to Cause Periapical Le- sions: Report of Two Cases}

\author{
Celia Elena Mendiburu-Zavala, Ricardo Peñaloza-Cuevas \\ and Rubén Cardenas Erosa
}

Department of Dentistry, Universidad Autónoma de Yucatán, Mérida, Yucatán, Mexico

\begin{abstract}
The basic pathogenic process that develops due to various causes which produce pulp or periapical pathology is the inflammatory response. The mechanical irritants such as occlusal trauma, direct release chemical mediators in the connective tissue of the dental pulp to initiate the process of inflammation as a defense mechanism that occurs in response to mechanical aggression. When it is not removed the etiological factor of long duration (occlusal trauma), aseptic pulp necrosis begins by destruction of the microvascular cells, lymphatic and nerve fiber systems. Subsequently, the products of the necrotic pulp could pass the apical region, as well as the inflammatory and immune dynamic process producing the apical periodontitis resulting in the release of cytokines such as interleukin 17 that is causing the activation of osteoclasts which produce bone resortion and enlargement and spreading of periapical lesions. Based on the above stated, is presented two cases of young healthy male of 13 and 22 years who were diagnosed by the endodontist professional with periapical pathology, which was possible caused by occlusal trauma. These cases eliminating the occlusal trauma and performing root canal treatment have a good prognosis.
\end{abstract}

Keywords: Occlusal trauma; Periapical lesions; Prognosis

\section{Introduction}

The condition or clinical entity known as occlusal trauma is synonymous with occlusion trauma, traumatic occlusion, traumatogenic occlusion, periodontal traumatism, occlusal overload, among others [1].

Marcos Lopez mentions that periapical pathologies can be caused by dental trauma affecting both the coronal portion as the tooth root. Occlusal alterations as bruxism, malocclusion and occlusal overload can trigger periapical damage [2].

*Corresponding author: Celia Elena Mendiburu-Zavala, Department of Dentistry, Universidad Autónoma de, Calle 25 No. 84 north between 60 and 20, Fracc Loma Bonita, Xcumpich, 97205 Mérida, Yucatán, Mexico, Tel: +52 9991955533 +52 9992923184; E-mail: cel_mendi@hotmail.com; mzavala@correo.uady.mx

Citation: Mendiburu-Zavala CE, Peñaloza-Cuevas R, Erosa RC (2016) Occlusal Trauma May Be a Factor to Cause Periapical Lesions: Report of Two Cases. J Dent Oral Health Cosmesis 1: 007.

Received: August 05, 2016; Accepted: November 04, 2016; Published: November 17, 2016
A study of 28 samples of pulp and the periodontal ligament of healthy premolars which the extraction was indicated for orthodontic reasons was done. Before extraction, was performed an experimentally induced occlusal trauma with occlusal interference by placing a block of resin on the occlusal surface, asking the patient to chew gum for 30 minutes. The remaining healthy premolars were extracted without occlusal trauma and served as controls. All samples were processed and the expression of Substance $\mathrm{P}(\mathrm{SP})$ was measured by radioimmunoassay [3]. Substance P is a decapeptide of Arg-Pro-Lys-ProGln-Gln-Fen-Fen-Gly-Leu-Met-NH2 structure, especially involved in pain perception. Nociceptive fibers are particularly common in the trigeminal nerve, which innervates the periapical tissues and dental pulp, where it has detected the presence of substance P [4]. The results with the occlusal trauma teeth experimentally induced were $45 \%$ and $120 \%$ greater expression of SP in the dental pulp and periodontal ligament respectively. The paired t-test showed statistically significant differences for both the human dental pulp and periodontal ligament ( $\mathrm{P}=0.02$ and $\mathrm{P}<0.001$, respectively) of the teeth under occlusal trauma when compared with the values of the control group, concluding that expression of SP is increased in human periodontal ligament and dental pulp [3].

When an existing chronic apical periodontitis is secondarily infected by bacteria produces what is called a "phoenix abscess". Symptoms and signs contain swelling, spontaneous and intense throbbing pain character, pain to percussion and palpation. The purulent exudate initially located below the periosteum, being able to pierce, producing diffuse inflammation, which if not treated in time, can form a sub mucosal or subcutaneous abscess [5].

\section{Reports}

\section{Ethical aspect}

Having a minor involved, it was asked the mother's informed and voluntary consent for her child to be taken pictures and published the data of his case. In the second case, the young adult man participant was asked for his approval, consent informed and voluntary for the same scientific order [6,7].

\section{Case 1}

A male 13 years old presents facial asymmetry in the left side (Figure 1), showing an area of inflammation. The mother referred that the patient's medical history was without any complications (no external trauma in the facial or iatrogenic factors done by a dental professional because it was de first time he came to the dentist) through the moment and before. To the anamnesis he referred severe, continuous and spontaneous pain in the upper left first premolar.

To the clinical inspection, it was observed that in any of the teeth of arches, dental cavities, tooth-plaque or some restoration (Figure 2). It was observed gingiva color, thickness and texture of normal appearance. Survey was conducted, which showed depth normal periodontal sulcus, without the presence of periodontitis. He was asked to occlude with articulating paper of 80 microns, with low thickness, marking premature contact point on the palate cusp of it (Figure 3), referring a severe and continuous pain when he occluded. It was called an 
Citation: Mendiburu-Zavala CE, Peñaloza-Cuevas R, Erosa RC (2016) Occlusal Trauma May Be a Factor to Cause Periapical Lesions: Report of Two Cases. J Dent Oral Health Cosmesis 1: 007.

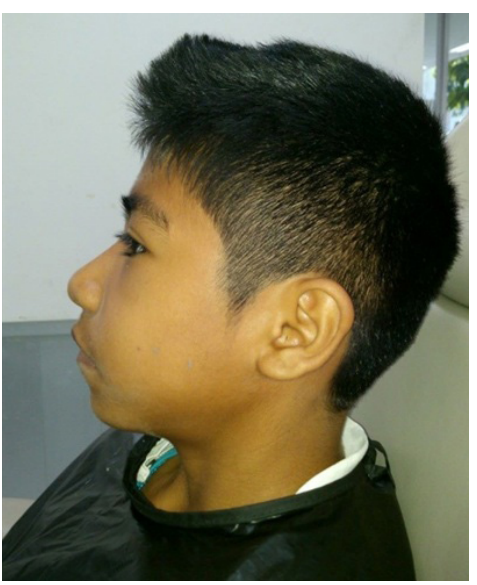

Figure 1: Left facial asymmetry.

occlusion expert to check the case, who verified that the child had occlusion trauma by identified the premature and balance contacts by manual palpation (Fremitus) and visualization of occlusal contacts during clenching in habitual intercuspation, lateral and protrusive movements of the mandible.

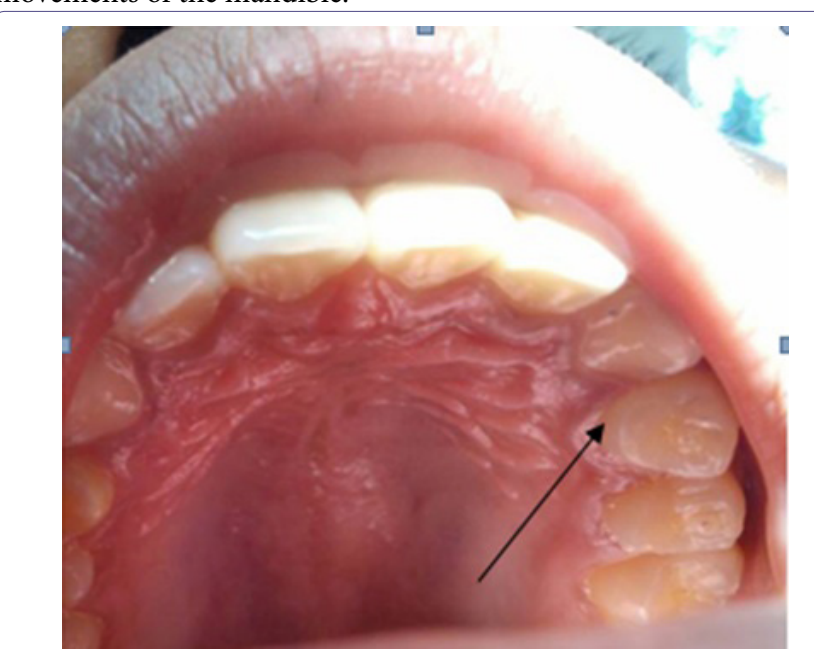

Figure 2: The tooth number 12, without the presence of caries, periodontal disease, restoration, crack or fracture.

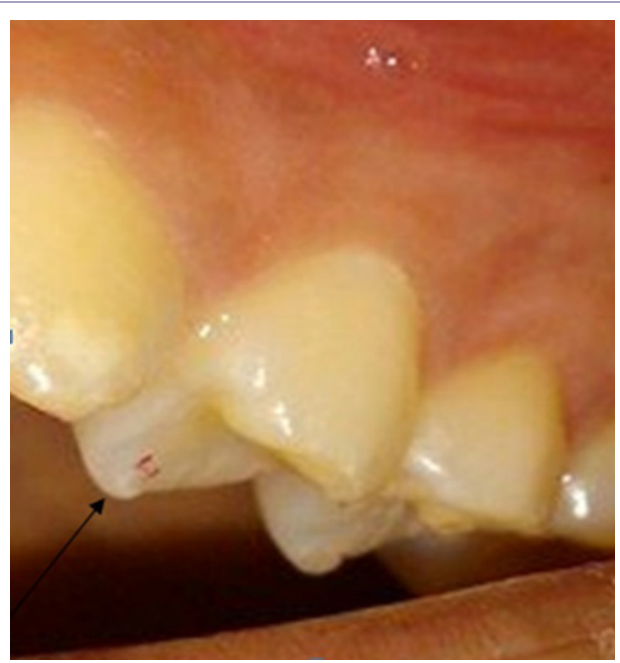

Figure 3: Occlusal marking.
Periapical radiograph was taken, showing a radiolucent periapical image portion of the tooth support bone resorption and a thickened periodontal ligament (Figure 4). The diagnosis was chronic apical periodontitis which acute, showing signs and symptoms characteristic of an acute periapical abscess or "phoenix abscess". A panorex and bitewing $\mathrm{x}$-rays were not taken, because the patient could not afford these diagnosis tools.

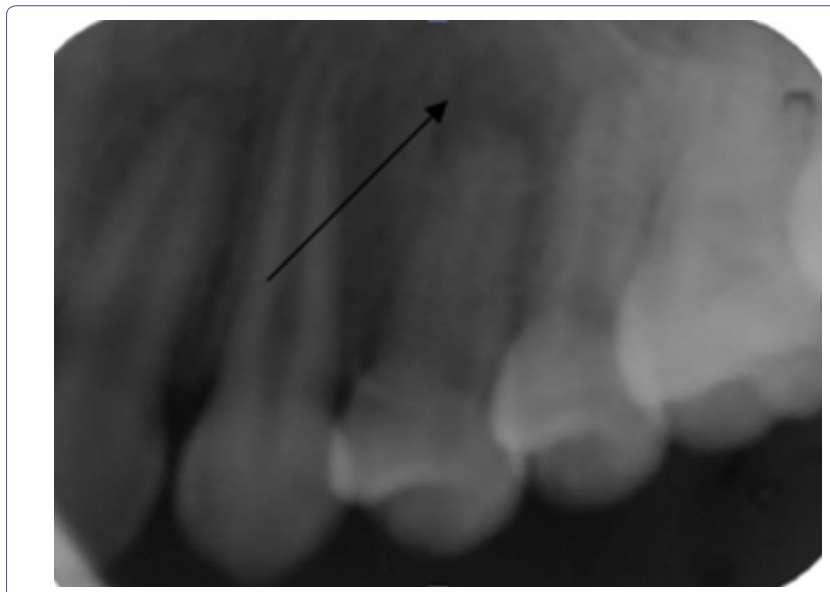

Figure 4: Periapical radiolucent portion of the first upper left premolar.

A selective wear points to relieve premature contacts and occlusal overload were done. For discomfort was prescribing paracetamol. After two days, it started root canal therapy. The sealing (Figure 5) was done 8 days after the symptoms (pain and malaise) stopped.

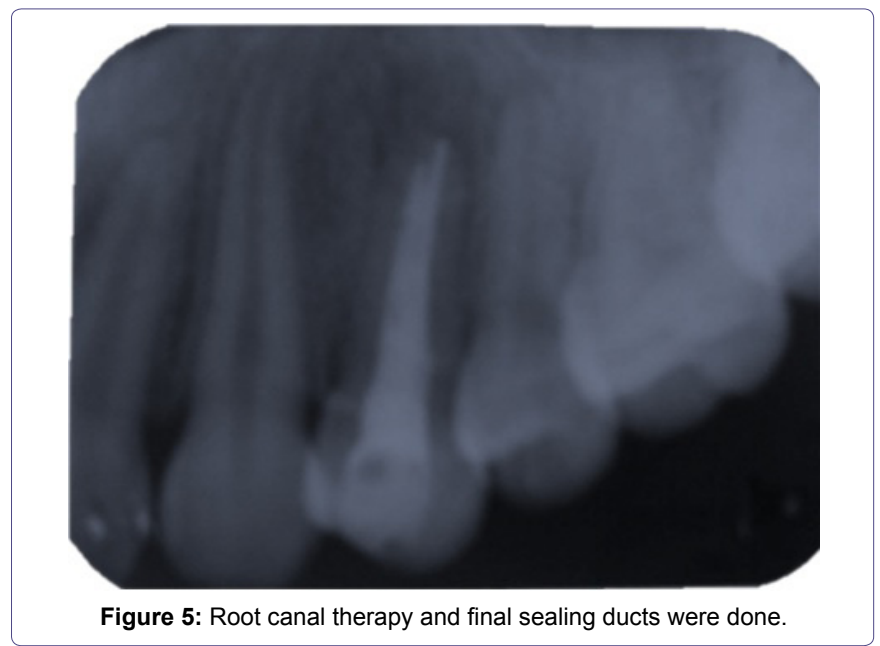

Case 2

A 22-year-old male was presented with severe pain intensity, facial edematous in appearance in the lower right portion. Through the anamnesis he referred, to consult the dentist not finding tooth with decay or periodontal disease. He was referred to the physicians for testing cabinet with the following results: Hematocrit 49.10, hemoglobin 16.60, platelets 307.00, WBC 7.60, VDRL, Brucella, Chagas, Hepatitis B and C and HIV, all negative. They decided to begin with drugs: Diclofenac sodium $100 \mathrm{mg}$, one tablet every $8 \mathrm{hrs}$; Paracetamol $500 \mathrm{mg}$, one tablet every $6 \mathrm{hrs}$; Procaine benzylpenicillin 600,000 IU, one every 24 hrs for 5 days, intramuscular route. Three days using the drugs, he referred not to have any relief and feel worse than before, so the doctor prescribed Dicloxacillin sodium $500 \mathrm{mg}$, one tablet every $8 \mathrm{hrs}$. For 5 days and metronidazole $500 \mathrm{mg}$, one tablet every 8 hours 
for 5 days. However, no pain relief and the inflammation of the face persist. Aching and scared for his condition, he decided to visit an endodontist.

Clinical inspection was done in the area concerned: Inflammation observed lower right side of the face. On palpation of cervical nodes on the right side, felt swollen compared to the opposite side. In teeth, no cavities, restoration, fracture, micro fracture or periodontitis was found. However, there was a mandibular lingual torus in the bicuspid right area. To clinical tests as percussion, showed severe, acute, full-length lower right second premolar and to palpation felt sensitive and a lump at the bottom of the bag in the same area. He was asked to occlude with articulating paper of 80 microns, marking premature contact point on the buccal cusp of that tooth, referring a severe and continuous pain when he occluded. It was used the same method as the child to see if he had occlusal trauma.

To assist the diagnosis, was taken a periapical radiography showing a periapex radiolucency image in the lower right second premolar, consistent with chronic apical periodontitis. On the other hand, the nature of pain, swelling and general discomfort was suspected of an acute periapical abscess or "phoenix abscess". However, a panorex and bitewing $\mathrm{x}$-rays were not taken, because of the costs for the patient.

It was stared with the root canal therapy because he was in a lot of pain. It was anesthetized the inferior alveolar nerve, isolating and when it was open to the entrance of the duct, it started to have an abundant drainage of purulent exudate (Figure 6), which mitigated his pain.

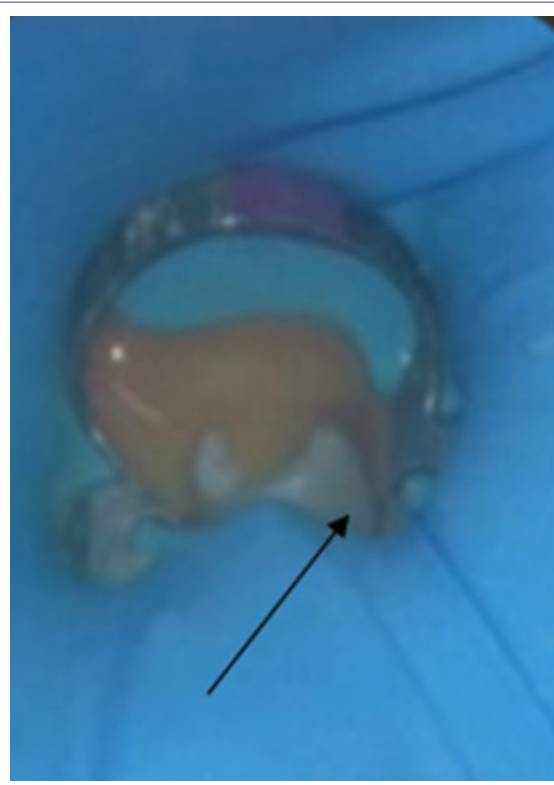

Figure 6: Drainage of purulent exudate.

It was proceeded to wash with abundant and great caution with sodium hypochlorite at $5 \%$. It was allowed to drain, but it did not stop the draining and knowing that it is not advisable to leave the access open with a cotton swab, it had to be done, not having another option in that moment. He was appointment for the next day, in which the root canal was washed, instrumented slightly, dried and introduced intracanal medication (calcium hydroxide) for 8 days. He was invited to continue taking the medicines that the physicians gave to him. After 8 days, he returned quieter without edema, and was proceeding to do the root canal treatment and in the next appointment, it was sealed (Figure 7)

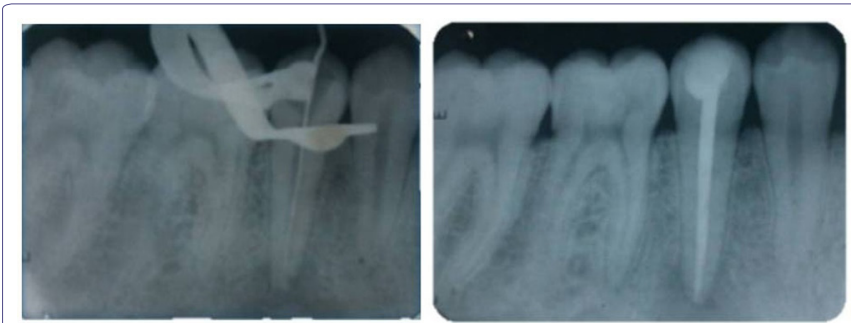

Figure 7: Root canal therapy and final sealing ducts.

\section{Discussion}

This report presents two cases of acute periapical abscess or "phoenix abscess" in which the occlusal trauma was probably the cause of this disease. Rupprecht [8], declared that the occlusal traumatism is the overall process by which a traumatogenic occlusion produces injury in the periodontal attachment apparatus. Also, he mentioned that occlusal adjustment or selective grinding is defined as reshaping the occluding surfaces of teeth by grinding to create harmonious contact relationships between the upper and lower teeth, which it was done in these two cases.

In the second case was shown a mandibular internal torus in the bicuspid right area, which is consistent with that reported by GarcíaGarcía et al., in which the mandibular torus could be found on the internal side of the horizontal branch of the jaw, above the mylohyoid line and at the level of the premolar and canine area. The exostoses origin is unclear, but approximately $70 \%$ was attributed to environmental factors, mainly related to occlusal stress [9].

So, it is important to make the differential diagnosis of a localized abscess involving a single tooth, including the acute apical abscess from the products of the necrotic toot [10] that could pass to the apical region, as well as the inflammatory and immune dynamic process producing the apical pathologist resulting in the release of cytokines such as Interleukin 17 that is causing the activation of osteoclasts which produce bone resortion and enlargement and spreading of periapical lesions. Consequently, this infection has the potential to develop into a true medical emergency if not treated promptly and aggressively [10]. Zhang et al., cited by Venkatesh et al., demonstrated that compressive force stimulates gene expression in the IL-17 and IL-17 (IL-17R) in MC3T3-E1 cells, and also results in the induction of osteoclastogenesis [11], which can be seen in the $\mathrm{x}$-rays imagines of these periapical areas of both cases.

The root canal therapy is the most indicated in these cases. The prognosis is good. Unfortunately, in underdeveloped countries such as Mexico, the public dentistry institutions do not have all the necessary equipment (it is limited to what they can buy with the money they received), the patients do not cooperated for their diagnosis and when are relieved of pain and endodontic therapy is completed, they do not return to their appointments. It is probably, because of the lack of financial resources, time or uninterested for final rehabilitation. Most dental medical education is suggested, so that these young people, who have dental problems at an early age, do not lose teeth at such a young age, which it is translated into adulthood with serious problems of steric, phonetics, occlusion, and nutrition.

\section{References}

1. Consolaro A (2012) Occlusal trauma cannot be compared to orthodontic movement. Dental Press J Orthod 17: 5-12.

2. Marcos JFL (2004) Etiología, clasificación y patogenia de la patología pulpar y periapical. Med Oral Patol Oral Cir Bucal 9: 52-62. 
3. Caviedes Bucheli J, Azuero Holguin MM, Correa Ortiz JA, Aguilar Mora MV Pedroza Flores JD, et al. (2011) Effect of Experimentally Induced Occlusa Trauma on Substance P Expression in Human Dental Pulp and Periodontal Ligament. J Endod 37: 627-630.

4. López Elizarraráz B, Aldape Barrios BC (2006) Principios del dolor dental. Rev Mex de Algol 3: 19-24.

5. Aponte-Rendon R, Teran I, Meza M, Greco-Machado Y (2010) Exacerbación aguda de una lesión crónica: absceso fénix. Endodoncia 28: 147-152.

6. World Health Organization (2009) Research ethics committees: Basic concepts for capacity-building. World Health Organization, Geneva, Switzerland.

7. Comité de Ética en Investigación en la Frontera México-Estados Unidos (2010) Prácticas y Protocolos Actuales, Comisión de Salud Fronteriza México-Estados Unidos.
8. Rupprecht D (2004) Trauma from occlusion: A review. Clinical Update 26: 25-27.

9. García-García AS, Martínez-González JM, Gómez-Font R, Soto-Rivadeneira $A$, Oviedo-Roldán $L(2010)$ Current status of the torus palatinus and torus mandibularis. Med Oral Patol Oral Cir Bucal 15: 353-360.

10. Edwards PC, Kanjirath P (2010) Recognition and management of common acute conditions of the oral cavity resulting from tooth decay, periodontal disease, and trauma: An update for the family physician. J Am Board Fam Med 23: $285-294$

11. Venkatesh N, Prasad M, Gowri SS, Vivek RG (2015) A review of root resorption in orthodontics. Annals and Essences of Dentistry 7: 23-27. 